\title{
Satisfacción de los estudiantes ante una sesión de exploración cardiológica impartida por compañeros entrenados
}

\author{
M. Cristina Rodríguez-Díez, Gregorio Mañeru-Zunzarren, Miguel Artaiz-Urdaci, Marta Ferrer-Puga, \\ Iosune Baraibar-Argota, Francisco J. Lage-Fernández, Nieves Díez
}

Objetivo. Conocer la valoración de los estudiantes respecto a la simulación como herramienta de formación y la opinión sobre la figura del alumno instructor.

Sujetos y métodos. El taller se realizó en el Centro de Simulación y tuvo una duración aproximada de 90 minutos. En cada sesión estaba el alumno instructor con diez compañeros. Los alumnos instructores fueron formados previamente por un profesor de cardiología. Al finalizar la sesión, los estudiantes cumplimentaron un cuestionario de satisfacción anónimo que contenía preguntas relacionadas con el aprendizaje basado en la simulación y sobre la capacidad de sus compañeros para actuar como formadores.

Resultados. Se obtuvieron encuestas de 291 estudiantes: 150 de quinto y 141 de sexto curso (82,4\% y 71,2\% de los matriculados, respectivamente). Los datos obtenidos del cuestionario de satisfacción respecto a la metodología de aprendizaje obtuvieron puntuaciones entre 3,64 y 4,75 sobre 5. Las preguntas que valoraban la opinión acerca de los alumnos formadores obtuvieron valores entre 4,88 y 4,93 sobre 5 .

Conclusiones. La simulación es una herramienta docente complementaria muy valiosa. La participación de alumnos instructores podría ser una ayuda adicional en los talleres de simulación de exploración cardiológica.

Palabras clave. Educación médica. Estudiantes entrenados. Exploración cardiológica. Habilidades clínicas. Simulación. Simulador cardiológico Harvey.

\section{Student satisfaction in response to a scanning session in cardiology given to trained students}

Aim. To acknowledge the students evaluation on the simulation as a training tool and their opinion on the ability of students as trainers.

Subjects and methods. The workshop was held at the Simulation Center and lasted approximately 90 minutes. The student in charge of training had 10 peers in each session. Previously, these students were taught to become trainers by a professor in Cardiology. After the session, students completed an anonymously satisfaction survey with questions related to the simulation-based learning and the ability of their peers to act as trainers.

Results. We obtained surveys from 291 students, 150 from fifth year and 141 from sixth year students $(82.4 \%$ and $71.2 \%$ respectively of those enrolled). The data that we obtained from the satisfaction survey with reference to the learning methodology had a score between 3.64 and 4.75 out of 5 . The questions that assessed the students' opinions on the ability of their peers to act as trainers obtained values between 4.88 and 4.93 out of 5 .

Conclusions. Simulation is a valuable complementary teaching tool. Therefore, the participation of students as trainers could provide an additional assistance in the cardiological examination simulation workshops.

Key words. Cardiological examination. Clinical skills. Harvey cardiac patient simulator. Medical education. Simulation. Training by students.

\section{Introducción}

Para obtener el título de graduado en medicina, todo alumno debe saber realizar, rutinariamente y sin supervisión, determinadas competencias. Entre las que corresponden al sistema circulatorio está el 're- conocer mediante la exploración física la anormalidad en la palpación de pulsos, la auscultación cardíaca y su significado' [1]. Para alcanzar este objetivo, los docentes explican los conceptos teóricos en el aula y la exploración cardiológica se realiza en los pacientes de las consultas o ingresados en el hospital. En
Unidad de Educación Médica (M.C. Rodríguez-Díez, M. Ferrer-Puga, N. Díez); Centro de Simulación (N. Díez, G. Mañeru-Zunzarren); Alumno interno Centro de Simulación (I. Baraibar-Argota, F.J. LageFernández); Facultad de Medicina; Universidad de Navarra. Servicio de Cardiología; Clínica Universidad de Navarra (M. Artaiz-Urdaci). Pamplona, Navarra, España.

Correspondencia: Dra. María Cristina Rodríguez Díez. Unidad de Educación Médica. Facultad de Medicina. Universidad de Navarra. Irunlarrea, 1. E-31008 Pamplona (Navarra)

E-mail:

cristinard@unav.es

Agradecimientos:

A todos los estudiantes que participaron en el estudio.

Conflicto de intereses: No declarado.

Competing interests: None declared.

(C) 2015 FEM 
este sentido, la simulación aplicada a la educación médica puede ser una herramienta complementaria para contribuir a la formación de los estudiantes.

El simulador de paciente cardiológico Harvey ${ }^{\circledR}$ fue creado hace más de 40 años (The Gordon Center for Research in Medical Education; Universidad de Miami) [2]. El maniquí presenta 30 situaciones cardiológicas diferentes que integra en un programa multimedia. En cada patología específica se puede medir sobre el simulador la presión arterial, el pulso yugular, la onda de pulso, el pulso arterial, los movimientos precordiales y la auscultación cardíaca y pulmonar.

El simulador se puede utilizar en una clase tradicional dirigida por un profesor, en un grupo reducido de alumnos o como autoaprendizaje. En nuestro caso, nos planteamos la opción de llevar a cabo el aprendizaje con un modelo de formación entre compañeros, de modo que un alumno entrenado previamente guía el taller a un grupo reducido de estudiantes. Una característica que puede destacarse es que el alumno formador que asume el rol de ayudar al otro no se constituye en una figura de autoridad, sino que lo capacita para enseñar a mejorar en un procedimiento concreto.

El trabajo que presentamos se ha realizado con estudiantes de medicina en sus dos últimos cursos de licenciatura, que ya habían cursado la asignatura de cardiología, pero no tenían incluido el aprendizaje mediante la simulación en su plan de estudios.

Los objetivos principales de nuestro estudio fueron conocer la valoración de los estudiantes respecto a la simulación como herramienta de formación y la opinión sobre la figura del alumno formador.

\section{Sujetos y métodos}

En el curso académico 2011-2012 se ofreció a los 380 alumnos matriculados en $5 .^{\circ}$ y $6 .^{\circ}$ curso de medicina (182 y 198 alumnos, respectivamente) la participación voluntaria en un taller de exploración con el simulador de paciente cardiológico Harvey. La media de edad de los alumnos participantes fue de $22,80 \pm 0,82$ años, siendo el $65,3 \%$ mujeres y el $35,7 \%$ varones. Las sesiones fueron dirigidas por dos alumnos formadores, de $5 .^{\circ}$ curso, que ya habían cursado la asignatura de cardiología.

Los dos alumnos formadores eran alumnos internos recién incorporados al Centro de Simulación y todavía no tenían experiencia en el uso del simulador de paciente cardiológico Harvey.

La formación previa de los alumnos instructores en la utilización del simulador fue responsabilidad de un profesor de la asignatura de cardiología, con experiencia en el uso del simulador, quien explicó en una sesión de una hora los objetivos de aprendizaje a los alumnos instructores. Se trabajó la exploración cardiológica normal y también las patologías más importantes que debían repasar, entre ellas la estenosis aórtica, la insuficiencia aórtica, el prolapso mitral, la estenosis mitral, la insuficiencia mitral y la pericarditis. Posteriormente, los dos alumnos internos acudieron de forma individual aproximadamente durante ocho horas para adquirir destreza en el manejo del simulador.

El taller que los alumnos instructores impartieron a sus compañeros se realizó en el Centro de Simulación y tuvo una duración aproximada de 90 minutos. En cada sesión estaba el alumno formador con diez compañeros.

Al finalizar la sesión, los estudiantes cumplimentaron un cuestionario de satisfacción anónimo sobre el aprendizaje basado en la simulación y sobre la capacidad de sus compañeros para actuar como formadores, con las siguientes preguntas:

1. La utilización del simulador favorece la integración de los conocimientos teórico-prácticos.

2. Las patologías cardíacas que se han visto eran adecuadas a tu nivel de conocimiento.

3. La práctica te ha servido para mejorar el conocimiento de las patologías estudiadas.

4. Los casos presentados te parecieron interesantes.

5. Han mejorado tus habilidades para realizar la auscultación cardíaca.

6. El entrenamiento con Harvey aumentará tu confianza cuando explores a pacientes reales.

7. Esta metodología de aprendizaje debería incluirse en el plan docente de la asignatura de cardiología de forma obligatoria.

8. La duración de los talleres ha sido la adecuada.

9. Los talleres han estado bien organizados por parte del Centro de Simulación.

10. Los talleres comenzaron y terminaron a la hora prevista.

11. Grado de satisfacción general con el taller.

12. La relación del 'alumno formador' fue cordial y amable con todo el grupo.

13. El alumno que impartió el taller favoreció la participación.

14. La formación del alumno que impartió el taller era la apropiada.

15. El 'alumno formador' ha demostrado que ha preparado la sesión.

16. En el transcurso de la sesión ha habido oportunidad de preguntar y resolver dudas.

Los cuestionarios se respondieron siguiendo la es- 
cala numérica de Likert de cinco dígitos, siendo 1 la puntuación mínima y 5 la puntuación máxima. Además, se recogieron los comentarios libres que escribieron los estudiantes.

\section{Análisis estadístico}

El análisis de los datos se realizó como valor medio \pm desviación estándar de cada ítem en una escala numérica. El análisis estadístico se efectuó con pruebas paramétricas, $t$ de Student para muestras independientes. Se estableció un valor de $p<0,05$ como límite de significación estadística. El análisis estadístico se realizó con el programa SPSS v. 15.0.

\section{Resultados}

En la figura se presentan los datos de todos los alumnos respecto a la satisfacción con la metodología de aprendizaje, con la organización de la sesión y con el alumno instructor. Las puntuaciones más altas fueron las relacionadas con los alumnos formadores, que obtuvieron valores entre 4,88 y 4,93 sobre 5 .

En el análisis de los resultados de las encuestas de satisfacción respecto al taller y metodología de aprendizaje desglosado por curso (Tabla I), los alumnos de sexto puntuaron más alto que los de quinto en casi todas las cuestiones, observando diferencias estadísticamente significativas $(p<0,05)$ en siete de 11 preguntas. Sin embargo, respecto a la satisfacción acerca de los alumnos formadores (Tabla II) no hubo diferencias entre los cursos.

En los resultados del análisis por sexo no se objetivaron diferencias significativas.

\section{Discusión}

La educación médica basada en el uso de simuladores ha aumentado en los últimos años y sus beneficios son conocidos también en la auscultación cardíaca [3-8]. Esta necesidad viene determinada porque en el entorno asistencial las oportunidades de los estudiantes de explorar a pacientes reales son limitadas [9]. Además, la simulación permite el entrenamiento en una competencia sin causar molestia al enfermo y en un entorno seguro.

En nuestro trabajo, las puntuaciones más altas fueron las relacionadas con la valoración de los alumnos formadores, por lo que podemos decir que la formación entre iguales resultó un modelo de aprendizaje atractivo y motivador para los estudiantes. La cercanía y la confianza que les inspiraba su com-
Figura. Cuestionario de satisfacción respecto al taller, metodología de aprendizaje y alumnos formadores: valores medios. Escala numérica de Likert de cinco dígitos, siendo 1 la puntuación mínima y 5 la puntuación máxima. Las barras de fondo gris hacen referencia a las preguntas sobre satisfacción respecto al taller y metodología de aprendizaje (P1-P11) y las barras de fondo negro se refieren a las preguntas sobre los alumnos formadores (P12-P16). Las preguntas se detallan en el texto.

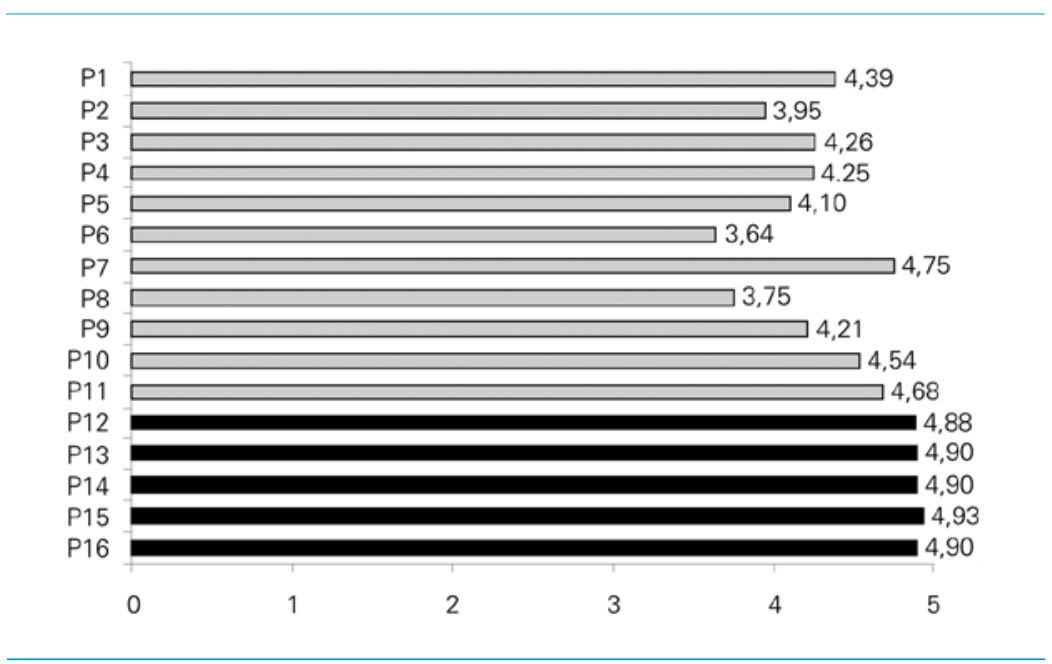

Tabla I. Satisfacción respecto al taller y metodología de aprendizaje según curso.

\begin{tabular}{lccc}
\hline & 5. curso $(n=150)$ & $6 .$. curso $(n=141)$ & $p$ \\
\hline Pregunta 1 & $4,29 \pm 0,797$ & $4,50 \pm 0,628$ & 0,010 \\
\hline Pregunta 2 & $3,79 \pm 0,985$ & $4,11 \pm 0,772$ & 0,003 \\
\hline Pregunta 3 & $4,14 \pm 0,875$ & $4,38 \pm 0,781$ & 0,013 \\
\hline Pregunta 4 & $4,17 \pm 0,839$ & $4,35 \pm 0,793$ & 0,060 \\
\hline Pregunta 5 & $3,89 \pm 0,984$ & $4,32 \pm 0,805$ & 0,000 \\
\hline Pregunta 6 & $3,59 \pm 0,998$ & $3,70 \pm 1,157$ & 0,362 \\
\hline Pregunta 7 & $4,71 \pm 0,630$ & $4,79 \pm 0,541$ & 0,203 \\
\hline Pregunta 8 & $3,53 \pm 1,224$ & $3,99 \pm 1,082$ & 0,001 \\
\hline Pregunta 9 & $4,17 \pm 0,932$ & $4,24 \pm 0,999$ & 0,550 \\
\hline Pregunta 10 & $4,63 \pm 0,727$ & $4,44 \pm 0,905$ & 0,046 \\
\hline Pregunta 11 & $4,63 \pm 0,630$ & $4,74 \pm 0,543$ & 0,108 \\
\hline
\end{tabular}

Escala numérica de Likert de cinco dígitos, siendo 1 la puntuación mínima y 5 la puntuación máxima (valores medios \pm desviación estándar). Las preguntas se detallan en el texto.

pañero los ayudó a participar e implicarse en la práctica. Por otra parte, creemos que todo el esfuerzo que realizaron los alumnos formadores, y así 
Tabla II. Satisfacción acerca de los alumnos formadores según curso.

\begin{tabular}{lccc}
\hline & 5. - curso $(n=150)$ & 6. o curso $(n=141)$ & $p$ \\
\hline Pregunta 12 & $4,89(0,310)$ & $4,87(0,363)$ & 0,477 \\
\hline Pregunta 13 & $4,91(0,292)$ & $4,89(0,332)$ & 0,722 \\
\hline Pregunta 14 & $4,91(0,282)$ & $4,89(0,353)$ & 0,598 \\
\hline Pregunta 15 & $4,92(0,296)$ & $4,94(0,298)$ & 0,643 \\
\hline Pregunta 16 & $4,91(0,314)$ & $4,89(0,340)$ & 0,600 \\
\hline
\end{tabular}

Escala numérica de Likert de cinco dígitos, siendo 1 la puntuación mínima y 5 la puntuación máxima (valores medios \pm desviación estándar). Las preguntas se detallan en el texto.

nos lo han manifestado, repercute en ellos mismos ayudándoles a mantener una actitud y disposición para continuar formándose. Nuestros resultados son acordes con otros trabajos que concluyen que la formación entre iguales es muy satisfactoria $[10,11]$. Un segundo paso sería demostrar que además, en estos talleres, se alcanzan los objetivos docentes marcados por el profesor y no sólo la satisfacción del alumno.

En cuanto a la valoración de la simulación como herramienta docente, los alumnos respondieron a la pregunta 'esta metodología de aprendizaje debería incluirse en el plan docente de la asignatura de cardiología de forma obligatoria' y le adjudicaron una puntuación de 4,75. Esta es una reflexión que consideramos muy adecuada y en el plan de estudios de grado en medicina ya se ha implementado un taller de exploración cardiológica con el simulador.

Sin embargo, las puntuaciones más bajas correspondieron a 'el entrenamiento con el simulador aumentará tu confianza cuando explores a pacientes reales', con una valoración de 3,64, y al ítem 'la duración de los talleres ha sido la adecuada,' con 3,75. Ambas valoraciones podrían estar relacionadas porque al aumentar la duración de los talleres, pensamos que también podría mejorar su confianza. Por tanto, un aspecto importante a mejorar es el aumento de la duración del taller y la posibilidad de que los alumnos puedan acudir en más ocasiones al Centro de Simulación.
Finalmente, aunque desconocemos el motivo por el que los alumnos de $66^{\circ}$ curso puntuaron más alto, podría deberse a que hayan intentado aprovechar más el taller porque en un breve plazo obtendrían el título de Licenciado en Medicina.

Queremos destacar como punto fuerte de este trabajo el gran número de estudiantes que asistieron a los talleres y de respuestas incluidas en los cuestionarios. Sin embargo, el punto débil es que no podemos demostrar que esta metodología influya en la adquisición de la competencia.

En conclusión, la enseñanza de la exploración cardiológica en un simulador impartida por estudiantes fue muy bien valorada, especialmente la participación de los alumnos instructores, y permitió incluir un gran número de asistentes en la práctica.

Bibliografía

1. Agencia Nacional de Evaluación de la Calidad y Acreditación (ANECA). Libro blanco. Título de grado en medicina. URL: http://www.aneca.es/var/media/150312/libroblanco_medicina _def.pdf.

2. Gordon MS. Cardiology patient simulator. Development of an animated manikin to teach cardiovascular disease. Am J Cardiol 1974; 34: 350-5.

3. Sullivan M, Nyquist J, Etcheverry J, Nally M, Scaff P, Abbottt A, et al. The development of a comprehensive school-wide simulation-based procedural skills curriculum for medical students. J Surg 2010; 67: 309-15.

4. Kneebone R. Evaluating clinical simulations for learning procedural skills: a theory-based approach. Acad Med 2005; 80: 549-53.

5. Issenberg SB, McGaghie WC, Gordon DL, Symes S, Petrusa ER, Hart IR, et al Effectiveness of a cardiology review course for internal medicine residents using simulation technology and deliberate practice. Teach Learn Med 2002; 14: 223-8.

6. Martínez G, Guarda E, Baeza R, Garayar B, Chamorro G, Casanegra P. Enseñanza de auscultación cardíaca a estudiantes y residentes de medicina mediante el uso de un simulador de ruidos cardíacos. Rev Esp Cardiol 2012; 65: 1134-42.

7. Fraser K, Wright B, Girard L, Tworek J, Paget M, Welikovich L, et al. Simulation training improves diagnostic performance on a real patient with similar clinical findings. Chest 2011; 139: 376-81.

8. Butter J, McGaghie W, Cohen ER, Marsha EK, Wayne DB. Simulation-based mastery learning improves cardiac auscultation skills in medical students. J Gen Intern Med 2010; 25: 780-5.

9. Scalese RJ, Obeso VT, Issenberg SB. Simulation technology for skills training and competency assessment medical education. J Gen Intern Med 2008; 23: 46-9.

10. Fernández-Prada M, González-Cabrera J, Ortega-López Y, Martínez-Bellón M, Fernández-Crehuet M, Bueno-Cavanillas A. Evaluación de un taller práctico sobre higiene de manos impartido por estudiantes entrenados. Educ Med 2012; 15 : 149-54.

11. Tolsgaard MG, Gustafsson A, Rasmussen MB, Hoiby P, Muller CG, Ringsted C. Students teachers can be as associate professors in teaching clinical skills. Med Teach 2007; 29: 553-7. 